\section{Rituximab in people living with HIV affected by immune-mediated renal diseases: a case-series}

\author{
G Alfano ${ }^{1,2}\left(\mathbb{D}, \mathrm{F}_{\text {Giaroni' }}\right.$, F Fontana ${ }^{2}$, L Neri $^{3}$, G Mosconi ${ }^{3,4}$, \\ C Mussini ${ }^{4}$, G Guaraldi ${ }^{4}$ and G Cappelli ${ }^{1,2}$
}

International Journal of STD \& AIDS 0(0) 1-6

(C) The Author(s) 2020 Article reuse guidelines: sagepub.com/journals-permissions DOI: I0.1 I77/0956462420946662 journals.sagepub.com/home/std (S)AGE

\begin{abstract}
Over the last two decades, rituximab (RTX) has played an important role in the treatment of some lymphoproliferative malignancies and immune-mediated diseases. RTX administration is generally safe and well-tolerated, but side effects including late-onset neutropenia, hypogammaglobulinemia, hepatitis B reactivation and rare cases of progressive multifocal leukoencephalopathy have been observed after its administration. Although there are no absolute contraindications regarding its use in people living with HIV (PLWH), the prescription of this drug has been principally limited in patients with oncohematological diseases. In this report, we described the outcome of four PLWH who underwent RTX therapy after the diagnosis of immune-mediated renal disease. The main RTX-associated adverse effects were leukopenia, late-onset neutropenia and decline of CD4+ and CD8+ T-cell counts. In addition, two of the four patients experienced pneumonia requiring hospitalization within six months from the last RTX infusion. We suggest that RTX should be used with caution in PLWH until further evidence emerges on its safety profile in this vulnerable population.
\end{abstract}

\title{
Keywords
}

HIV, rituximab, antiretroviral therapy, kidney transplantation, neutropenia, rejection

Date received: 19 October 2019; accepted: 9 July 2020

\section{Introduction}

Rituximab (RTX) is a monoclonal chimeric antibody used to target the antigen CD20 expressed on both normal and neoplastic B-cells. Originally, it was included in non-Hodgkin lymphoma and chronic lymphocytic leukemia therapies, whereas now it has also been approved to treat several autoimmune diseases such as rheumatoid arthritis, anti-neutrophil cytoplasmic autoantibody (ANCA)-associated vasculitis and pemphigus vulgaris. ${ }^{1}$ In the last decade, off-label use of RTX has been extended to immune-mediated renal diseases including cryoglobulinemia-associated nephropathy, membranous glomerulonephritis (MGN) and lupus erythematosus. In addition, the promising results of RTX in induction immunosuppressive therapy, desensitization programs and treatment of antibodymediated rejection have broadened its therapeutic application in the field of kidney transplantation. ${ }^{2,3}$ The mechanism of action of drug is only partially understood. Besides the rapid depletion of pre-B and mature B-cells expressing CD20 with subsequent decrease of antibody production, RTX has an immunomodulatory effect on both CD4+ and CD8+ T-cells. ${ }^{4-6}$

Unfortunately, the beneficial effects of RTX-based therapy are offset by a series of unwanted adverse effects including late-onset neutropenia, ${ }^{7-9}$

\footnotetext{
'Surgical, Medical and Dental Department of Morphological Sciences, Section of Nephrology, University of Modena and Reggio Emilia, Modena, Italy

${ }^{2}$ Nephrology Dialysis and Transplant Unit, University Hospital of Modena, Modena, Italy

${ }^{3}$ Nephrology and Dialysis Unit, Hospital of Forli-Cesena, Forli-Cesena, Italy

${ }^{4}$ Clinic of Infectious Diseases, University Hospital of Modena, Modena, Italy
}

Corresponding author:

G Alfano, Section of Nephrology, University Hospital of Modena and Reggio Emilia, Via del Pozzo, 7I 4II 24 Modena, Italy.

Email: gaetano.alfano@unimore.it 
hypogammaglobulinemia ${ }^{10}$ and probably an early decrease in CD4+ T-cell count. ${ }^{11-14}$ RTX seems also associated with an increasing rate of infectious complications due to transient immunosuppression. ${ }^{15-20}$ Recently, Trivin et al. ${ }^{21}$ reported that in a retrospective cohort of 98 patients treated with RTX for glomerulopathy, 25 of them $(21.6 \%)$ experienced infectious complications, most frequently bacterial in origin.

The tendency of RTX to increase the susceptibility to infections has likely limited its use in people living with HIV (PLWH). As a result, the drug has been principally used as chemotherapeutic agent to treat lymphoproliferative diseases including non-Hodgkin lymphoma ${ }^{22}$ and Castleman disease. ${ }^{23}$ The available information about safety of RTX from these sources is scarce and rather conflicting. Indeed, whereas a randomized trial comparing R-CHOP (RTX plus cyclophosphamide, doxorubicin, vincristine and prednisone) with the CHOP regimen showed a higher occurrence of fatal infections in patients treated with $\mathrm{RTX}^{24}$ a recent observational study found no differences in the rate of death for infections in patients who received RTX for HIV-associated lymphoma. ${ }^{25}$ In light of these opposing results and the little information available on RTX in PLWH, we report our experience in the use of RTX to target B-cells involved in the pathogenesis of immune-mediated renal diseases, focusing on its safety profile.

\section{Cases presentation}

\section{Case I}

A 65-year-old Caucasian woman with a hisstory of HIV-1 infection since 1996, hypertension, Helicobacter pylori infection-associated gastritis, uterine prolapse and depressive disorder developed nephrotic syndrome in January 2017.

On presentation, laboratory tests were notable for serum creatinine of $1.4 \mathrm{mg} / \mathrm{dl}$, albumin $2.2 \mathrm{mg} / \mathrm{dl}$ and spot urine protein/creatinine ratio of $8.3 \mathrm{mg} / \mathrm{mg}$. She had a normal CD4+ T-cells count $(1070$ cells/ $\mu$ l) with undetectable HIV-1 viral load (VL) ( $<40$ copies). Antiretroviral therapy (ART) consisted of three-drug combination therapy with lamivudine, efavirenz and abacavir.

The renal biopsy performed revealed a histological pattern compatible with MGN (stage 2), confirmed by serum levels of anti-phospholipase-A2-receptor autoantibodies (anti-PLA2R) of $237.64 \mathrm{UR} / \mathrm{ml}$.

The patient was to receive intravenous (IV) infusion of RTX $375 \mathrm{mg} / \mathrm{m}^{2}$ once weekly for four consecutive weeks in July 2017 without antimicrobial prophylaxis.

After three doses of RTX administered at a dosage of $750 \mathrm{mg}$, the patient experienced moderate neutropenia ( 770 vs. 3250 cells $/ \mu \mathrm{L}$ ) in the absence of fever or other signs of infections. A single dose of granulocyte colony-stimulating factor (G-CSF) was used to raise the neutrophil count that reached a normal value (3260 cells $/ \mu \mathrm{L}$ ) after 19 days from its nadir. After RTX treatment, HIV VL remained unchanged, but T CD4+ cell count decreased slightly to 611 cells/ $\mu$, even though it remained in the reference range throughout the observation period. Despite the decrease of antiPLA2R antibody levels $(1.9 \mathrm{UR} / \mathrm{ml})$, proteinuria remained steadily in the nephrotic range. In December 2017, worsening renal function (creatinine $2.63 \mathrm{mg} / \mathrm{dl}$ vs. a baseline creatinine of $1.5 \mathrm{mg} / \mathrm{dl}$ ) prompted a course of second-line immunosuppressive therapy consisting of steroids and cyclosporine. In March 2018, she was admitted for fever and dyspnea due to pneumonia that was treated with a course of antibiotic therapy. At the end of one-year follow-up, renal function remained stable (creatinine $1.76 \mathrm{mg} / \mathrm{dl}$, eGFR $30 \mathrm{ml} / \mathrm{min}$ ) in the absence of proteinuria.

\section{Case 2}

A 24-year-old African woman living with HIV underwent kidney transplantation in 2010 after four years spent on dialysis for end-stage renal disease (ESRD) secondary to HIV-associated nephropathy. Her medical history included also multinodular goitre. Her immunosuppression consisted of everolimus in combination with reduced-dose cyclosporine whereas ART was based on dolutegravir and rilpivirine. According to our protocol, prophylaxis against cytomegalovirus and Pneumocystis carinii infection was discontinued at 6 and 12 months after transplantation, respectively.

In May 2017, her post-transplant course was complicated by a slight increase in serum creatinine $(1.5 \mathrm{mg} /$ $\mathrm{ml}$ vs. a baseline creatinine of $1.3 \mathrm{mg} / \mathrm{ml}$ ) associated with proteinuria greater than $400 \mathrm{mg} / \mathrm{dl}$.

Renal biopsy revealed a histological pattern compatible with antibody-mediated transplant rejection. The diagnosis was confirmed by the detection of a donorspecific antibody (DSA) titer of 9068 mean fluorescent intensity (MFI).

In light of these histological findings, a course of immunosuppressive therapy including three boluses of methylprednisolone and plasmapheresis was started. Additionally, IV infusion of RTX $375 \mathrm{mg} / \mathrm{m}^{2}$ once weekly for four consecutive weeks was administered in order to reduce antibody production, and maintenance immunosuppressive therapy was potentiated with the switch from cyclosporine and everolimus to tacrolimus and mycophenolic acid. Prophylaxis with trimethoprim-sulfamethoxazole was administered for six months. 
After 3.1 months from the last RTX dose, a fall in leukocyte and neutrophil counts was seen with a nadir of 220 neutrophils $/ \mu \mathrm{L}$ without infectious sequelae. Four doses of G-CSF restored the neutrophil count $(2200$ cells $/ \mu \mathrm{L})$ three days after its nadir. In April 2019, despite the disappearance of DSAs, a second graft biopsy was performed for elevation in the serum creatinine concentration $(2.7 \mathrm{mg} / \mathrm{dl})$ associated with significant proteinuria ( $1.5 \mathrm{~g} /$ daily). Histological evaluation identified progression to the chronic stage of the underlying humoral rejection.

\section{Case 3}

A 54-year-old African woman living with HIV-2 infection underwent kidney transplantation in 2012 after 12 years spent on dialysis for ESRD caused by malignant hypertension. She also had a medical history of type 2 diabetes mellitus and tertiary hyperparathyroidism. Her immunosuppression consisted of everolimus in combination with reduced-dose cyclosporine, mycophenolic acid and steroids. ART was based on lamivudine, maraviroc and raltegravir. HIV VL was $<40$ copies and T-CD4+ cell count was 1080 cells $/ \mu 1$.

Post-transplant was complicated by two episodes of biopsy-proven T-cell-mediated graft rejection that were successfully treated with a short course of high-dose corticosteroid.

In August 2013, a rapid increase in serum creatinine level from baseline $1.8 \mathrm{mg} / \mathrm{ml}$ to $2.78 \mathrm{mg} / \mathrm{dl}$ prompted renal graft biopsy.

The histological evaluation showed a pattern of antibody-mediated rejection that was confirmed by a high titer of DSA ( $>10,000$ MFI both class I and II).

A course of combined immunosuppressive therapy including three boluses of methylprednisolone, four cycles of IV immunoglobulins and a single dose of $700 \mathrm{mg}$ of RTX was administered. The poor experience of RTX in PLWH limited further infusions of the drug. Prophylaxis against Pneumocystis jirovecii pneumonia for six months started after the administration of antirejection therapy. After five months from RTX treatment, neutrophils reached a nadir of 230 cells $/ \mu \mathrm{L}$ in the absence of signs of systemic infection. Treatment with G-CSF and mycophenolic acid withdrawal resulted in a normalization of the WBC count one month later. CD4+ T-cell count reached a nadir of 256 cells $/ \mu 1$ a few days after RTX treatment but returned to over 800 cells $/ \mu$ l one month after. Despite DSA becoming negative and HIV VL remaining undetectable, a relentless worsening of renal function continued in the following months and the patient started hemodialysis in May 2014.

\section{Case 4}

A 43-year-old Caucasian man living with HIV since 2013 underwent kidney transplantation in 2016 after three years spent on peritoneal dialysis for ESRD caused by MGN. His medical history also included failure of a living-donor kidney transplant, HCV infection, hyperthyroidism, and previous syphilis, human herpesvirus 6 and 7 infections. His immunosuppressive therapy was a combination of tacrolimus, mycophenolic acid and steroid, whereas ART was based on dolutegravir and rilpivirine.

Two years after kidney transplant, the patient had a decline in kidney function (serum creatinine raised from a baseline of $1.7 \mathrm{mg} / \mathrm{dl}$ to $2.6 \mathrm{mg} / \mathrm{dl}$ ) in the absence of HIV replication ( $<40$ copies).

A graft biopsy was performed. Detection of high levels of DSA ( $>10,000$ MFI for class II) and histologic evidence of $\mathrm{C} 4 \mathrm{~d}$ staining and chronic tissue injury lead to the diagnosis of chronic antibody-mediated rejection.

A course of immunosuppressive therapy consisting of a single dose of IV RTX (750 mg) and IV immunoglobulin $(0.1 \mathrm{~g} / \mathrm{kg})$ was administered in October 2019.

After one week from the second infusion of RTX $(750 \mathrm{mg})$, the patient developed severe Legionella pneumonia manifesting with acute dyspnoea and fever. RTX therapy was discontinued and the infection was effectively treated with levofloxacin without sequelae. In March 2019, his CD4+ T-cell count dropped below 100 cells $/ \mu \mathrm{L}$ and prophylaxis against Pneumocystis carinii pneumonia was started. DSA showed a moderate decline but renal function worsened quickly reaching a pre-dialysis stage with a creatinine value of $5.17 \mathrm{mg} / \mathrm{dl}$ in April 2019.

\section{Discussion}

This case-series showed that RTX should be used cautiously in PLWH because it is associated with some serious post-treatment side effects that may increase the risk of infections. We described the outcome of four PLWH who were treated with RTX after the diagnosis of renal disease triggered by immune-mediated mechanisms. RTX was used as single agent for the treatment of idiopathic MGN in a non-transplanted subject and in combination with other immunosuppressive agents for the treatment of antibody-mediated graft rejection in three kidney transplant recipients (Table 1). RTX was administered uniformly at dose of $375 \mathrm{mg} / \mathrm{m}^{2}$ once weekly with the total number of infusions varying from one to four per patient. The difference in RTX dose prescription was ascribed to the early onset of significant adverse effects leading to RTX withdrawal (case 1 and 4) and to the prudential 
Table I. Main laboratory and clinical findings in people living with HIV who underwent rituximab therapy.

\begin{tabular}{|c|c|c|c|c|}
\hline & Case I & Case 2 & Case 3 & Case 4 \\
\hline Age (years) & 65 & 24 & 54 & 43 \\
\hline Immune-mediated kidney disease & MGN & AMR & AMR & AMR \\
\hline Total number RTX infusions & 3 & 4 & $\mathrm{I}$ & 2 \\
\hline RTX dose at each infusion (mg) & 750 & 700 & 700 & 750 \\
\hline \multicolumn{5}{|l|}{ Leucocytes count (cells/ $\mu \mathrm{L})$} \\
\hline Pre-RTX & 8890 & 5280 & 7450 & 8590 \\
\hline Nadir post-RTX & 3336 & 1720 & 2120 & 3390 \\
\hline \multicolumn{5}{|l|}{ Lymphocyte count (cells $/ \mu \mathrm{L}$ ) } \\
\hline Pre-RTX & 2820 & 1600 & 1900 & 1470 \\
\hline Nadir post-RTX & 2140 & 760 & 1004 & 220 \\
\hline \multicolumn{5}{|l|}{ Neutrophil count (cells/ $\mu \mathrm{L}$ ) } \\
\hline Pre-RTX & 3250 & 2240 & 4420 & 4500 \\
\hline Nadir post-RTX & 770 & 220 & 230 & 2800 \\
\hline Months from RTX to neutropenia* & 0.7 & 3.1 & 4.8 & 4.7 \\
\hline \multicolumn{5}{|l|}{ Platelet count $\left(10^{3}\right.$ cells $\left./ \mu \mathrm{L}\right)$} \\
\hline Pre-RTX & 244 & 360 & 391 & 186 \\
\hline Nadir post-RTX & 206 & 298 & 167 & 153 \\
\hline \multicolumn{5}{|l|}{ CD4+ T-cell count (cells/ $\mu \mathrm{L})$} \\
\hline Pre-RTX & 1070 & 758 & 1080 & 888 \\
\hline Nadir post-RTX & 611 & 539 & 256 & 66 \\
\hline \multicolumn{5}{|l|}{ CD8+ T-cell count (cells/ $\mu \mathrm{L})$} \\
\hline Pre-RTX & $|32|$ & 573 & 891 & 554 \\
\hline Nadir post-RTX & 1286 & 385 & 262 & 59 \\
\hline \multicolumn{5}{|l|}{$\lg G / \lg M / \lg A(\mathrm{mg} / \mathrm{dl})$} \\
\hline Pre-RTX & $526 / 142 / 117$ & $1310 / 268 / 158$ & $797 / 168 / 200$ & $539 / 68 / 216$ \\
\hline Nadir post-RTX & $244 / 106 / 94$ & III3/208/I79 & $587 / 65 / 167$ & $483 / 20 / 142$ \\
\hline G-CSF administration & Yes & Yes & Yes & No \\
\hline Post-RTX infectious complications & Pneumonia & None & None & Pneumonia \\
\hline
\end{tabular}

Abnormal lab test results were considered secondary to the pharmacological effect of rituximab if they occurred within six months from the last rituximab infusion and other potential causes (e.g., infections, drugs, plasmapheresis, etc.) were excluded.

AMR: antibody-mediated rejection; RTX: rituximab; G-CSF: granulocyte colony-stimulating factor; MGN: membranous glomerulonephritis; Ig: immunoglobulin.

use of RTX as a new antirejection drug in HIV $+\mathrm{KT}$ recipients (case 3). However, Despite the reduced dosage compared to the standard protocol (once weekly for four consecutive weeks), RTX consistently decreased the level of serum anti-PLA2R antibodies and DSA, serological markers of MGN and antibody-mediated rejection, respectively.

In our patients, RTX was associated with clinically relevant adverse effects such as leukopenia, neutropenia, transient decline in CD4+ and CD8+ T-cell count that potentially increased the susceptibility to infections (Table 1). After RTX therapy, we recognized a decline in the neutrophil count which was clinically relevant only in three patients (75\%). Neutropenia developed after a period ranging from 0.7 to 4.8 months from the first RTX infusion in the absence of evident signs of infection or sepsis. Neutropenia was responsive to the administration of G-CSF in all three cases.

Two patients experienced bacterial pneumonia that necessitated hospital admission. In both cases, pneumonia was treated with intravenous antibiotics and resolved without sequelae. We also noticed a tendency toward the reduction of CD4+ and CD8+ T-cell count within 6 months from RTX therapy. Unfortunately, we have few data to trace all changes in immunoglobulin levels in these patients. However, the decline of serum immunoglobulin levels was moderate and probably multifactorial.

Late-onset neutropenia is a delayed adverse effect of RTX. The incidence varies between 3 and $27 \%{ }^{26}$ and it seems to occur more frequently among patients treated for hematological cancers ${ }^{27}$ than autoimmune diseases. ${ }^{28}$ The mechanism by which RTX determines neutropenia is still unclear. It has been suggested that the aberrant reconstitution of B-cells after therapy cessation may disrupt neutrophil homeostasis through formation of anti-neutrophils antibodies or production of chemokines. There is compelling evidence supporting the theory of polymorphism in the immunoglobulin $(\mathrm{Ig}) \mathrm{G} \quad \mathrm{Fc}$ receptor $(\mathrm{Fc} \gamma \mathrm{R})$. 
Notably, subjects harboring the specific polymorphism in the IgG Fc receptor $F C G R 3$ position $158 \mathrm{~V} / \mathrm{F}$ have a higher incidence of RTX-induced neutrophilia. ${ }^{21}$ Likely RTX interacts with high-affinity FCGR3 158 on neutrophils to induce a high apoptotic response in these cells. Late-onset neutropenia remains an unpredictable event and occurs after a median of 38-175 days from last RTX dose. ${ }^{26}$ The severity of neutropenia is variable, but in case of neutropenic fever, G-CSF may be given to enhance the recovery of neutrophils.

In the literature, there are no data about the incidence of RTX-induced neutropenia in PLWH. In this group of patients, the drug has been mainly used for HIV-associated lymphoproliferative disease ${ }^{23,29}$ and, to a lesser extent, for rejections in solid organ transplant, ${ }^{20,30-33}$ anti-glomerular basal membrane disease $^{34}$ and autoimmune thrombocytopenia. ${ }^{23}$ The majority of information regarding safety and tolerability of the drug has been gleaned from trials on patients with hematologic malignancies where an alarmingly high rate of fatal infections has been found. ${ }^{29,35}$ Only a single episode of fatal progressive multifocal leukoencephalopathy was reported following therapy with RTX $^{19}$ in one heart transplant recipient.

It is unclear if PLWH are more prone to developing RTX-related side effects compared to other subsets of the population. CD20 lymphopenia probably aggravates CD4 lymphopenia, and consequently, can worsen the state of immunodeficiency. However, in absence of further randomized trials comparing RTX with other chemotherapy or immunosuppressive regimens, it is difficult to weigh the effects of RTX implicated in late-onset neutropenia and infectious complications because several confounding factors may be involved in this cohort of patients. First, the exposition to combination chemotherapy does not allow us to differentiate the effect of each single agent. Second, the use of protease inhibitors may increase the risk of chemotherapy-induced neutropenia. ${ }^{35}$ Lastly, low CD4 T-cell count $(<450$ cells $/ \mu \mathrm{L})$, a condition commonly found in PLWH, is a risk factor for infections and early death. ${ }^{36}$

The principal limitation of our study is the small number of patients that does not allow us to generalize our findings. Further studies should be carried out in a much broader cohort of PLWH in order to clearly weigh the risks and benefits of RTX therapy in this population. When the use of RTX is inevitable, we cautiously suggest avoiding the drug in neutropenic patients with altered CD4 T-cell count. Given the risk of delayed adverse effects, close follow-up is warranted with frequent and careful monitoring of blood count, CD4+ and CD8+ T-cell counts until further evidence is found on the infectious risk associated with RTX therapy.

\section{Conclusions}

In our series, RTX induced serious laboratory abnormalities that may have increased the susceptibility to infections. Based on this observation, the use of this drug deserves special attention in PLWH. Close follow-up with careful monitoring of complete blood count, CD4+ and CD8+ T-cells - even a long-time after RTX therapy cessation - is needed in all PLWH undergoing RTX therapy in order to identify and potentially treat life-threatening infections.

\section{Authors' contributions}

Gaetano Alfano and Francesco Giaroni have contributed equally to this work.

\section{Declaration of conflicting interests}

The author(s) declared no potential conflicts of interest with respect to the research, authorship, and/or publication of this article.

\section{Funding}

The author(s) received no financial support for the research, authorship, and/or publication of this article.

\section{ORCID iD}

G Alfano (D) https://orcid.org/0000-0003-0591-8622

\section{References}

1. Nixon A, Ogden L, Woywodt A, et al. Infectious complications of rituximab therapy in renal disease. Clin Kidney J 2017; 10: 455-460.

2. Djamali A, Kaufman DB, Ellis TM, et al. Diagnosis and management of antibody-mediated rejection: current status and novel approaches. Am J Transplant 2014; 14: 255-271.

3. Weiner GJ. Rituximab: mechanism of action. Semin Hematol 2010; 47: 115-123.

4. Guo J, Fujiyoshi A, Willcox B, et al. Increased aortic calcification is associated with arterial stiffness progression in multiethnic middle-aged men. Hypertension 2017; 69: 102-108.

5. Stasi R, Poeta GD, Stipa E, et al. Response to B-cell-depleting therapy with rituximab reverts the abnormalities of T-cell subsets in patients with idiopathic thrombocytopenic purpura. Blood 2007; 110: 2924-2930.

6. Fukuno K, Tsurumi H, Ando N, et al. Late-onset neutropenia in patients treated with rituximab for nonHodgkin's lymphoma. Int J Hematol 2006; 84: 242-247.

7. Arai Y, Yamashita K, Mizugishi K, et al. Risk factors for late-onset neutropenia after rituximab treatment of B-cell lymphoma. Hematology 2015; 20: 196-202.

8. Monaco WE, Jones JD and Rigby W. Rituximab associated late-onset neutropenia - a rheumatology case series and review of the literature. Clin Rheumatol 2016; 35: 2457-2462. 
9. Karmacharya P, Poudel DR, Pathak R, et al. Rituximabinduced serum sickness: a systematic review. Semin Arthritis Rheum 2015; 45: 334-340.

10. McLaughlin P, Grillo-López AJ, Link BK, et al. Rituximab chimeric anti-CD20 monoclonal antibody therapy for relapsed indolent lymphoma: half of patients respond to a four-dose treatment program. J Clin Oncol 1998; 16: 2825-2833.

11. Saville MW, Benyunes MC and Multani PS. No clinical evidence for CD4+ cell depletion caused by rituximab. Blood 2003; 102: 408-409.

12. Lavielle M, Mulleman D, Goupille P, et al. Repeated decrease of CD4+ T-cell counts in patients with rheumatoid arthritis over multiple cycles of rituximab treatment. Arthritis Res Ther 2016; 18: 253.

13. Besada E and Nossent JC. CD4 cell count and CD4/CD8 ratio increase during rituximab maintenance in granulomatosis with polyangiitis patients. PeerJ 2016; 4: e2487.

14. Gea-Banacloche JC. Rituximab-associated infections. Semin Hematol 2010; 47: 187-198.

15. Kelesidis T, Daikos G, Boumpas D, et al. Does rituximab increase the incidence of infectious complications? A narrative review. Int $J$ Infect Dis 2011; 15: e2-e16.

16. Lanini S, Molloy AC, Prentice AG, et al. Infections in patients taking rituximab for hematologic malignancies: two-year cohort study. BMC Infect Dis 2013; 13: 317.

17. Kronbichler A, Kerschbaum J, Gopaluni S, et al. Trimethoprim-sulfamethoxazole prophylaxis prevents severe/life-threatening infections following rituximab in antineutrophil cytoplasm antibody-associated vasculitis. Ann Rheum Dis 2018; 77: 1440-1447.

18. Wallace ZS, Choi H and Stone JH. Risk of severe infection following rituximab and the efficacy of antimicrobial prophylaxis. Ann Rheum Dis 2020; 79: e40. annrheumdis-

19. Loyaga-Rendon RY, Taylor DO and Koval CE. Progressive multifocal leukoencephalopathy in a heart transplant recipient following rituximab therapy for antibody-mediated rejection. Am J Transplant 2013; 13 : 1075-1079.

20. Trivin C, Tran A, Moulin B, et al. Infectious complications of a rituximab-based immunosuppressive regimen in patients with glomerular disease. Clin Kidney J 2017; 10: 461-469.

21. Spina $M$ and Tirelli U. Rituximab for HIV-associated lymphoma: weighing the benefits and risks. Curr Opin Oncol 2005; 17: 462-465.

22. Noy A. HIV multicentric Castleman: rituximab sans maintenance. Blood 2017; 129: 2045-2046.

23. Kaplan LD, Lee JY, Ambinder RF, et al. Rituximab does not improve clinical outcome in a randomized phase 3 trial of CHOP with or without rituximab in patients with HIV-associated non-Hodgkin lymphoma: AIDS-Malignancies consortium trial 010. Blood 2005; 106: $1538-1543$.

24. Wyen C, Jensen B, Hentrich M, et al. Treatment of AIDS-related lymphomas: rituximab is beneficial even in severely immunosuppressed patients. AIDS 2012; 26: 457-464.

25. Wolach O, Bairey O and Lahav M. Late-Onset neutropenia after rituximab treatment. Medicine (Baltimore) 2010; 89: 308-318.

26. Grant C, Wilson WH and Dunleavy K. Neutropenia associated with rituximab therapy. Curr Opin Hematol 2011; 18: 49-54.

27. Salmon JH, Cacoub P, Combe B, et al. Late-onset neutropenia after treatment with rituximab for rheumatoid arthritis and other autoimmune diseases: data from the autoimmunity and rituximab registry. RMD Open 2015; 1: e000034.

28. Weng W-K, Negrin RS, Lavori P, et al. Immunoglobulin G Fc receptor FcgammaRIIIa $158 \mathrm{~V} / \mathrm{F}$ polymorphism correlates with rituximab-induced neutropenia after autologous transplantation in patients with nonHodgkin's lymphoma. J Clin Oncol 2010; 28: 279-284.

29. Moscoso-Solorzano GT, Baltar JM, Seco M, et al. Single dose of rituximab plus plasmapheresis in an HIV patient with acute humoral kidney transplant rejection: a case report. Transplant Proc 2007; 39: 3460-3462.

30. Touzot M, Pillebout E, Matignon M, et al. Renal transplantation in HIV-infected patients: the Paris experience. Am J Transplant 2010; 10: 2263-2269.

31. Waheed S, Sakr A, Chheda ND, et al. Outcomes of renal transplantation in HIV-1 associated nephropathy. PLoS ONE 2015; 10: e0129702.

32. Alfano G, Mori G, Fontana F, et al. Clinical outcome of kidney transplantation in HIV-infected recipients: a retrospective study. Int J STD AIDS 2018; 29: 1305-1315.

33. Wechsler E, Yang T, Jordan SC, et al. Anti-glomerular basement membrane disease in an HIV-infected patient. Nat Clin Pract Nephrol 2008; 4: 167-171.

34. Junagade PS, Height S, Ruiz G, et al. Rituximab in children with autoimmune thrombocytopenia complicating underlying congenital or acquired immunodeficiency state. Blood 2006; 108: 3977-3977.

35. Bower M, McCall-Peat N, Ryan N, et al. Protease inhibitors potentiate chemotherapy-induced neutropenia. Blood 2004; 104: 2943-2946.

36. Borg C, Ray-Coquard I, Philip I, et al. CD4 lymphopenia as a risk factor for febrile neutropenia and early death after cytotoxic chemotherapy in adult patients with cancer. Cancer 2004; 101: 2675-2680. 